UVX 2008 (2009) 113-118

(C) EDP Sciences, 2009

DOI: $10.1051 / \mathrm{uvx} / 2009018$

\title{
Characterization of the FLASH XUV-FEL pulses by two-color photoionization experiments
}

\author{
M. Meyer ${ }^{1}$, D. Cubaynes ${ }^{1}$, D. Glijer ${ }^{1}$, S. Düsterer², W.B. Li ${ }^{2}$, A. Azima², \\ H. Redlin ${ }^{2}$, J. Feldhaus ${ }^{2}$, J. Dardis ${ }^{3}$, P. Hayden ${ }^{3}$, P. Hough ${ }^{3}$, V. Richardson ${ }^{3}$, \\ E.T. Kennedy ${ }^{3}$, J.T. Costello ${ }^{3}$, R. Taïeb ${ }^{4}$, A. Maquet ${ }^{4}$, E.V. Gryzlova ${ }^{5}$, \\ S.I. Strakhova ${ }^{5}$ and A.N. Grum-Grzhimailo ${ }^{1,5}$
}

${ }^{1}$ LIXAM, UMR 8624, CNRS - Université Paris Sud, Bâtiment 350, 91405 Orsay Cedex, France

${ }^{2}$ HASYLAB at DESY, Notkestr. 85, 22607 Hamburg, Germany

${ }^{3}$ National Center for Plasma Science and Technology and School of Physical Sciences, Dublin City University, Dublin 9, Ireland

${ }^{4}$ UPMC, Université Paris 06, CNRS, UMR 7614, LCPMR, 11 rue Pierre et Marie Curie, 75231 Paris Cedex 05, France

${ }^{5}$ Institute of Nuclear Physics, Moscow State University, Moscow 119991, Russia

\begin{abstract}
The current key performance indicators of the pump-probe facility at the Free Electron Laser in Hamburg (FLASH) are described. The temporal and spatial characteristics of both the extreme ultraviolet (XUV) Free Electron Laser and the temporally synchronized optical femtosecond Ti:Sapphire laser are determined by measuring two-color above threshold ionization in rare gases. Characteristic sidebands appear in the photoelectron spectrum under the combined action of both radiation fields providing a very sensitive probe for the spatial and temporal overlap of both pulses. The high spatial acceptance of a magnetic bottle electron spectrometer enabled us to obtain single-shot photoelectron spectra and to correct for the inherent time jitter between both light sources, which reduces the temporal resolution when recording spectra in average mode. As an illustration of the first application, experimental results on polarization control in the two-photon two-color ionization of atomic He are presented.
\end{abstract}

\section{INTRODUCTION}

In the last years, experimental studies of photoinduced processes using short wavelength radiation have gained a strong stimulus by the advent of Free Electron Lasers (e.g. [1]) operating on the principle of self-amplified spontaneous emission (SASE) (e.g. [2]). In particular, the start of operation of the Free electron LASer in Hamburg (FLASH) at DESY in mid-2005 has opened up many fascinating possibilities for new experimental studies [3], which make use of ultra-short laser pulses at high fundamental photon energies $(13-48 \mathrm{~nm})$ with high peak and average power [4,5]. For pump-probe experiments and time-resolved studies the XUV radiation from FLASH can be combined with an optical laser, which provides femtosecond infra-red $(120 \mathrm{fs}, 800 \mathrm{~nm})$ and picosecond visible $(12 \mathrm{ps}, 523 \mathrm{~nm})$ pulses, electronically synchronized with the Free Electron Laser pulses (temporal width 10-20 fs). The optical laser can be used to prepare species in a particular, well-defined state prior to ionization in the XUV field or it can produce a strong dressing field during the photoionization process giving rise to additional structures in the electron spectrum [6,7], i.e. causing changes to the ionization dynamics. In addition, by making use of the wavelength tunability of both sources, one or both excitation steps can be made resonant [8]. Numerous two-color pump-probe experiments, involving intense laser fields of very different photon energies and focussing on photoionization and molecular dissociation processes, can be explored in this way. But in order to make full use of the two independent femtosecond sources, the 

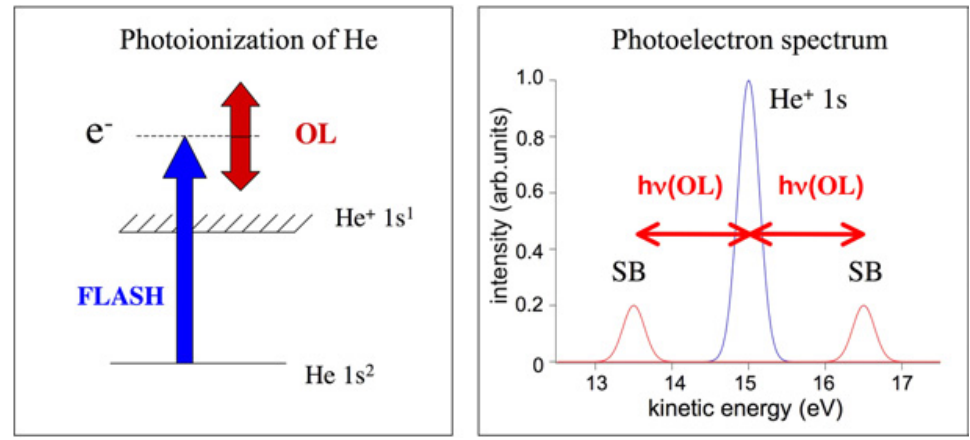

Figure 1. Scheme of the above threshold ionization (ATI) processes giving rise to the sidebands in the photoelectron spectrum. Sidebands (SB) occur only at spatial and temporal overlap between the pulses of the FEL and the optical laser $(\mathrm{OL})$

synchronization and the spatial and temporal stability of the set-up has to be characterized under real experimental conditions.

A powerful tool to obtain information about the temporal overlap of femtosecond pulses is provided by the process of two-color Above Threshold Ionization (ATI) (Fig. 1). This technique is based on photoelectron spectroscopy of rare gas atoms to analyze the photoionization signal produced by an XUV radiation field in the presence of a strong optical laser beam. Upon photoionization by the XUV photons, the electrons can emit or absorb one or more additional light quanta from the intense optical dressing field $\left(>10^{10} \mathrm{~W} / \mathrm{cm}^{2}\right)$. This process is observed as a sideband structure on both sides of the main atomic line in the photoelectron spectrum [6,7]. Intensity and shape of these sidebands depend strongly on the temporal and spatial profiles of the two femtosecond pulses [7] and can therefore be utilized to characterize the XUV pulses $[9,10]$. Since the underlying physical processes are theoretically well understood [11], the method can even be applied and extended to the analysis of single-shot spectra allowing for each individual event to determine the time delay between both the XUV and the optical laser pulses [12].

\section{EXPERIMENTAL SET-UP}

Details of the two-color pump-probe set-up are given elsewhere [12,13]. Briefly, the XUV Free Electron Laser (FEL) and the optical laser beams were introduced into the vacuum chamber in a collinear geometry and intersected an effusive gas jet within the acceptance angle of a magnetic bottle electron spectrometer (MBES) (Fig. 2). The FEL was operated at $25.5 \mathrm{~nm}(48.5 \mathrm{eV})$ and $13.8 \mathrm{~nm}(89.9 \mathrm{eV})$ at a repetition rate of $5 \mathrm{~Hz}$ in single bunch mode, pulse energies up to $50 \mu \mathrm{J}$, spectral bandwidth of $0.5-1 \%$ and pulse duration of 10-20 fs estimated from the spectral distribution $[4,5]$. The optical dressing field is produced by ultra-short optical laser pulses from an intense Ti-Sapphire laser (up to $2 \mathrm{~mJ}, 800 \mathrm{~nm}$, $120 \mathrm{fs}$ to $4 \mathrm{ps}$ ), which is synchronized to the reference frequencies $(1.3 \mathrm{GHz}$ and $108 \mathrm{MHz})$ from the master oscillator driving the electron accelerator.

During the experiments, the radiation of the optical laser was transported via a dedicated beam line over a distance of $30 \mathrm{~m}$ to the experimental vacuum chambers and was focussed there via an off-axis parabola ( $30^{\circ}$ off-axis, $f=272 \mathrm{~mm}, 76 \mathrm{~mm}$ diameter) to a focus diameter of about $50 \mu \mathrm{m}$. Optical dressing fields in the order of $10^{10}-10^{12} \mathrm{~W} / \mathrm{cm}^{2}$ were easily obtained in this geometry. The diameter of the FEL beam in the interaction region was determined to be about $30 \mu \mathrm{m}$, hence minimizing the effect of spatial jitter for the two-color experiments. Spatial alignment of both laser beams was achieved by monitoring, with the aid of a microscope, the spatial profiles of each beam on a phosphor screen placed at the interaction region of the MBES. The time delay between the two laser pulses was controlled by 


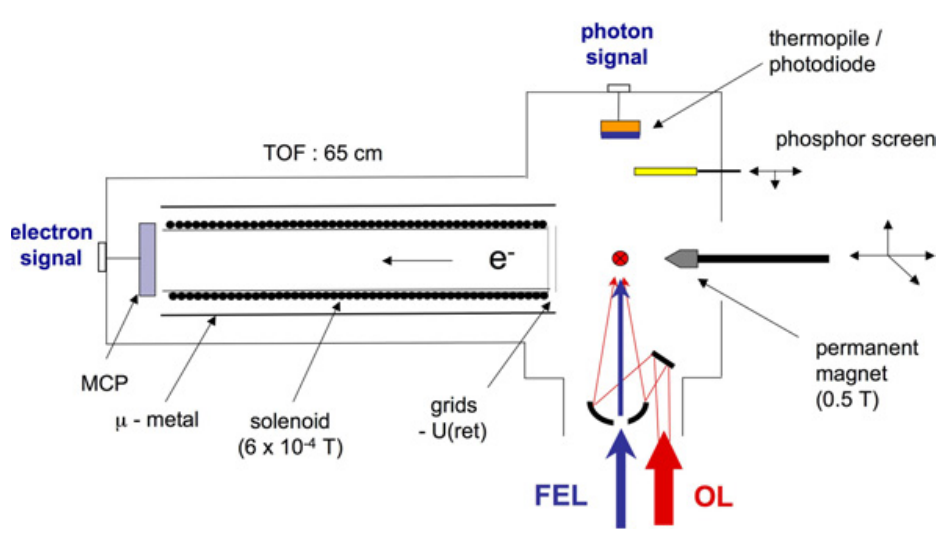

Figure 2. Experimental set-up for the pump-probe experiments using the combination of the FEL with an optical laser.

passing the optical beam through a variable delay stage. Long term drifts of up to \pm 3 ps between the two lasers were monitored by a fast streak camera with better than 1 ps (r.m.s.) resolution.

Electron spectra are recorded with the aid of a specially designed MBES $[10,14]$. The photoelectrons which are produced by the interaction of the XUV radiation with the effusive gas jet are directed by a strong permanent magnet $(0.5 \mathrm{~T})$ towards a $65 \mathrm{~cm}$ long flight tube. A solenoid producing a weak guiding field $(0.6 \mathrm{mT})$ brings almost all the electrons to a microchannel plate detector (MCP) and a time-of-flight (TOF) detection scheme allows the kinetic energies of all photoelectrons to be measured simultaneously. Due to the large collecting efficiency of the MBES, the set-up can be applied to low density targets and, in the case of the intense FEL beam, allows the analysis of the photoionization process on a shot-to-shot basis [12]. The best energy resolution of $1-2 \%$ of the kinetic energy is achieved by decelerating the electrons with the aid of electrostatic retardation fields at the entrance of the flight tube.

\section{EXPERIMENTAL RESULTS AND DISCUSSION}

\subsection{Two-color spectroscopy in average mode}

The high kinetic energy region of a series of two-color photoelectron spectra of atomic Xe is displayed in Fig. 3. The spectra were recorded at different fixed temporal delays $(\Delta \mathrm{T})$ between the pulses of the FEL and the optical laser by averaging over 250 single pulses. With FLASH operating at a wavelength of $25.5 \mathrm{~nm}$ (i.e. $48.6 \mathrm{eV}$ ), photoionization of the Xe $5 \mathrm{p}$ shell gives rise to the two strong lines at $E_{k i n}=36.5 \mathrm{eV}$ and $35.2 \mathrm{eV}$ attributed to the ionic states $X e^{+} 5 p^{5}{ }^{2} P_{3 / 2}$ and ${ }^{2} P_{1 / 2}$, respectively. The additional small lines at higher kinetic energy are the signature of the ATI process. These so-called sidebands in the photoelectron spectrum are caused by the simultaneous action of both the XUV and optical fields, and show up only in the region of strong temporal overlap, i.e. in the region close to $\Delta \mathrm{T}=0 \mathrm{fs}$. In the given example, where the optical dressing field was produced by the $800 \mathrm{~nm}$ pulses of about $20 \mu \mathrm{J}$ pulse energy and $120 \mathrm{fs}$ temporal width, sidebands up to 4th order are observable in the region of perfect overlap.

Since the sideband structure is very sensitive to the temporal (and spatial) overlap between the FEL and optical laser pulses, the measurements are used to determine the temporal resolution of the pumpprobe set-up at FLASH. On the right hand side of Fig. 3 the intensity of the sidebands is plotted as a function of the temporal delay $\Delta \mathrm{T}$. This cross-correlation curve can be fitted by a Gaussian profile with a full width at half maximum (FWHM) of about $600 \mathrm{fs}$. This value corresponds to the temporal resolution which is obtained in time-resolved two-color experiments without any further characterization or control of both pulses. Since the pulse widths of the FEL and the optical laser were determined to be about 

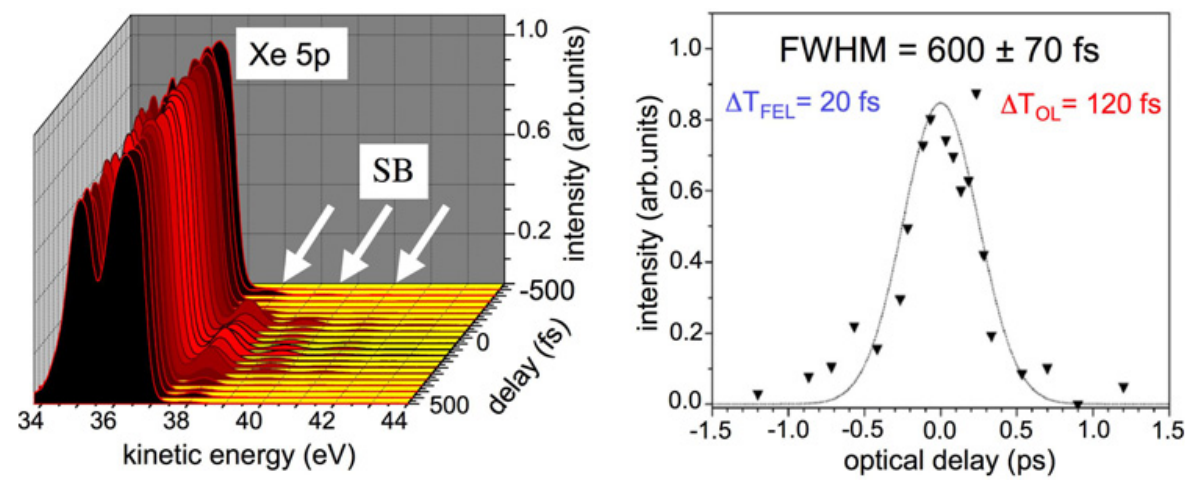

Figure 3. (Left) Part of the two-color photoelectron spectra of atomic Xe photoionized by the XUV radiation from FLASH $(25.5 \mathrm{~nm})$ in the presence of the optical laser. (Right) Cross-correlation curve for the two-color photoionization by FLASH (20 fs) and optical laser (120 fs) pulses.

10-20 fs [5] and $120 \mathrm{fs}$ [12], respectively, this temporal width is mainly caused by the inherent jitter of the SASE FEL. From our measurements, the jitter is determined to about 590 fs (FWHM) [12], which represents the dominant contribution for experiments recorded in the signal averaging mode.

\subsection{Two-color spectroscopy in single-pulse mode}

In order to improve the temporal resolution at the FLASH pump-probe facility and to overcome the limitations due to the temporal jitter of the FEL pulses, additional characterization of the pulses is necessary. For the case of the ATI measurements, we have taken advantage of the possibility to record a full photoelectron spectrum for each individual FEL pulse by using the MBES [13]. Such singleshot two-color photoionization spectra of atomic Xe, recorded at $\hbar \omega(\mathrm{FEL})=89.9 \mathrm{eV}(13.8 \mathrm{~nm})$ and at optimal temporal overlap ( $\Delta \mathrm{t}=0 \mathrm{fs}$ ), are shown in Fig. 4 . Even though recorded at the same nominal temporal delay, the selected spectra are markedly different with respect to the number of sidebands. Due to the jitter of about $590 \mathrm{fs}$, a single $20 \mathrm{fs}$ wide FEL pulse sometimes doesn't overlap at all with the 120 fs wide optical pulse (spectrum (d) without sideband structure) or is in perfect overlap (spectrum (a) with the maximum number of sidebands) or overlaps, but with a small delay with respect to the optical pulse (spectra (b) and (c) with fewer sidebands). Since the overall intensity of the photoionization process is determined by the number of FEL photons and the optical field only serves to redistribute the intensity on the kinetic energy scale, the one-color signal for the case of strongest sideband intensity, i.e. strongest overlap, is markedly decreased in the single-pulse mode. In the average spectra (cf. Fig. 3) this effect can hardly be seen due to the high number of events without overlap, which produce the dominant Xe $5 \mathrm{p}$ photolines.

The variation of the sideband intensity as a function of the position of the FEL pulse with respect to the broader optical pulse can be used to determine with much higher precision the temporal delay between both pulses, for cases where both are overlapping in time. The comparison to theoretical calculations [11], which take into account the different effective strengths of the optical dressing field at different temporal delays, reproduce very well the observed structures (Fig. 4). The spectra with one or two sidebands correspond to a decrease of dressing field intensity by a factor of 3 and 12 , respectively, i.e. a temporal delay of 80 and $115 \mathrm{fs}$ with respect to perfect overlap at $\Delta \mathrm{t}=0 \mathrm{fs}$. In this way, we demonstrate that it is possible to obtain via the analysis of the sideband intensity a determination of the relative temporal delay between individual independent FEL and optical pulses with a precision of better than $50 \mathrm{fs}$. We note that the region of temporal overlap can be increased by stretching the optical pulses at the cost of sideband signal intensity. However, we also note that with this analysis it is not possible to determine which of the pulses comes first. 

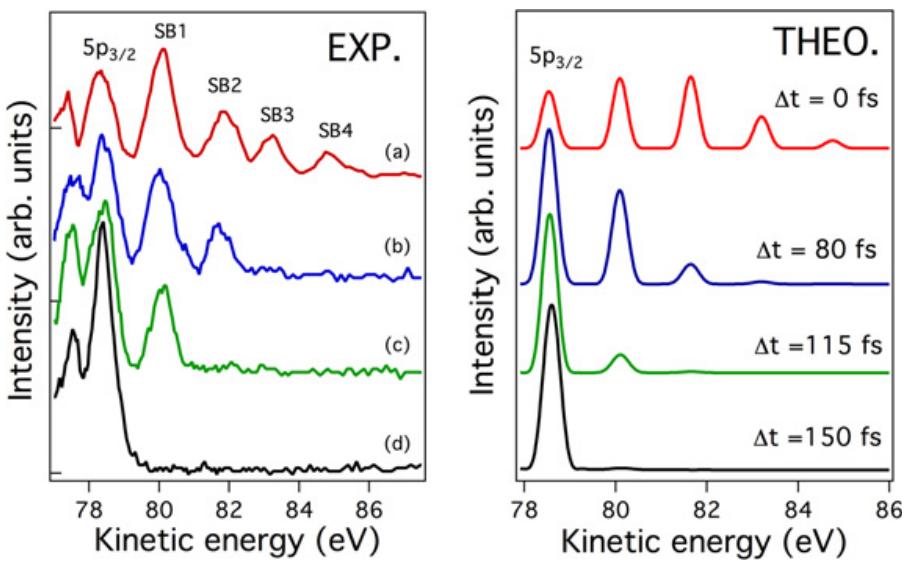

Figure 4. (Left) Two-color single-shot photoelectron spectra of atomic Xe photoionized in the $5 p$ shell by the XUV radiation from FLASH $(13.5 \mathrm{~nm})$ in the presence of the optical laser. (Right) Theoretical simulations of the Xe $5 p_{3 / 2}$ spin orbit component in the two-color photoionization spectrum for different temporal delays between the FEL (20 fs) and optical (120 fs) pulses.

\subsection{Polarization effects in the two-color photoionization of $\mathrm{He}$}

After having characterized the temporal characteristics of the two-color pump-probe set-up, we have used it for a first application, namely to determine the polarization dependence of the two-color above threshold ionization process in atomic He [15]. By taking advantage of the well-defined linear polarization of both laser beams, we have measured photoelectron spectra for different relative orientation $\theta$ between the polarization vectors of the FEL and the optical laser (Fig. 5). The strongest intensity of the sidebands and smallest of the He 1s main line are observed for parallel orientation $(\theta=$ $0^{\circ}$ ). Much less intensity is transferred to the sidebands, when the polarization vectors are perpendicular to each other $\left(\theta=+90^{\circ}\right.$ or $\left.-90^{\circ}\right)$. The amplitude of the intensity variation can be directly related to the ratio of the partial cross sections in the two-color photoionization process. When only a moderate dressing field is applied $\left(\approx 8 \times 10^{10} \mathrm{~W} / \mathrm{cm}^{2}\right)$ and only one sideband is observed in the photoelectron spectra, the variation can explicitly be described by

$$
\sigma(\theta) \propto 3 S_{2}+\left(5 S_{0}+S_{2}\right) \cos ^{2} \theta
$$

with $S_{i}=\left|T_{i}\right|^{2}$, where $T_{i}(i=0,2)$ are second-order radial integrals associated with the emission of $s$ - and $d$-electrons, respectively [16]. The measured contrast between maximal and minimal sideband intensity $\sigma_{\max } / \sigma_{\min }=3.4 \pm 0.5$ leads to an experimental ratio $S_{0}: S_{2}=1.25 \pm 0.3$. This ratio is in good agreement with the theoretical values of $S_{0}: S_{2}=0.98$ and 1.0 obtained within the secondorder perturbation calculation and the soft-photon approach, respectively, but deviates strongly from the predictions of the propensity rules [17], favoring in general transitions related to an increase of the angular momentum in the case of absorption.

\section{CONCLUSION}

Using the process of above threshold ionization (ATI) in rare gases, we have been able to characterize the temporal stability of the XUV pulses from the Free Electron Laser FLASH with respect to an external synchronized optical laser. When recording spectra by averaging over many pulses the effective temporal resolution was determined to about $600 \mathrm{fs}$ FWHM, which is nearly completely related to the temporal jitter of the FEL. Since the intensity of the sidebands in the photoelectron spectra provides a precise sensor for the strength of the dressing field, i.e. for the relative delay of the ionizing FEL pulse with 


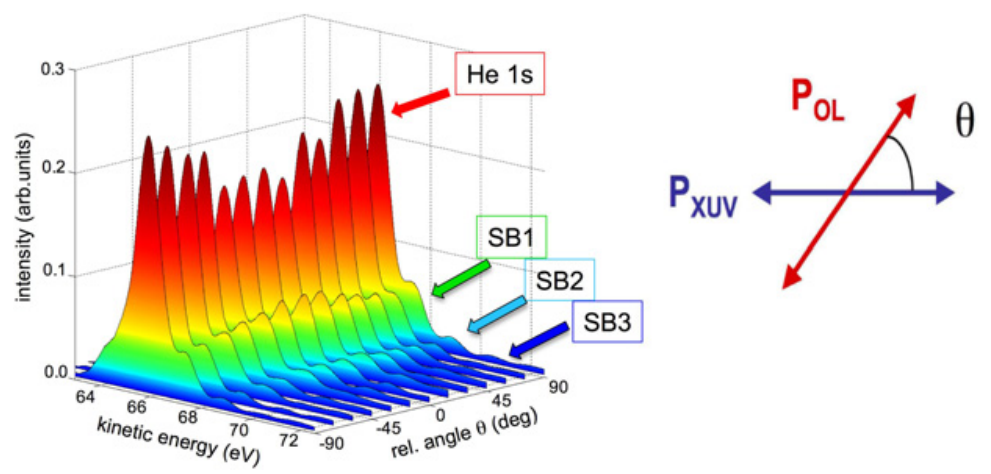

Figure 5. Two-color photoelectron spectra of atomic He photoionized by the XUV radiation from FLASH $(13.5 \mathrm{~nm})$ in the presence of the optical laser field $\left(6 \times 10^{11} \mathrm{~W} / \mathrm{cm}^{2}\right)$ displayed as a function of the relative angle $\theta$ between the linear polarization vectors of the XUV-FEL and optical laser (OL).

respect to the optical pulse, the temporal precision was improved to a value of about 50 fs for overlapping pulses by making use of a single shot analysis. Finally, as a first application the polarization effects in the two-color two-photon ionization of atomic He was measured providing for the He 1s photoionization a determination of the relative importance of the $s$ and $d$ continua.

\section{Acknowledgements}

We greatly appreciate the work of the scientific and technical team at FLASH, in particular the machine operators and run coordinators. Support from the EU RTD-project X-Ray FEL Pump-Probe HRPI-CT-1999-50009, from Science Foundation Ireland (Frontiers), IRCSET, the HEA PRTLI 4 INSPIRE programme and from the FranceIreland ULYSSES programme are acknowledged.

\section{References}

[1] J. Feldhaus, J. Arthur and J. B. Hastings, J. Phys. B 38, (2005) S799.

[2] E. Saldin, E. Schneidmiller and M. Yurkov, The Physics of Free Electron Lasers (Springer, New York 1999) Chap. 6, p. 353.

[3] C. Bostedt et al., Nucl. Instrum. Meth. (Kai-Siegbahn Memorial Volume), (2008) submitted.

[4] V. Ayvazyan et al., Eur. Phys. J. D 37, (2006) 297.

[5] W. Ackermann et al., Nat. Photon. 1, (2007) 336.

[6] J. M. Schins et al., Phys. Rev. Lett. 73, (1994) 2180.

[7] T. E. Glover, R. W. Schoenlein, A. H. Chin and C. V. Shank, Phys. Rev. Lett. 76, (1996) 2468.

[8] S. I. Themelis, P. Lambropoulos and M. Meyer, J. Phys. B 37, (2004) 4281.

[9] E. S. Toma et al., Phys. Rev. A 62, (2000) 061801.

[10] M. Meyer et al., Phys. Rev. A 74, (2006) 011401.

[11] A. Maquet and R. Taïeb, J. Mod. Opt. 54, (2007) 1847.

[12] P. Radcliffe et al., Appl. Phys. Lett. 90, (2007) 131108.

[13] P. Radcliffe et al., Nucl. Instrum. Meth. A 583, (2007) 516.

[14] J. Eland, O. Vieuxmaire, T. Kinugawa, P. Lablanquie, R. Hall and F. Penent, Phys. Rev. Lett. 90, (2003) 530031.

[15] M. Meyer et al., Phys. Rev. Lett. 101, 193002 (2008).

[16] R. Taïeb, V. Véniard, A. Maquet, N. L. Manakov, and S. I. Marmo, Phys. Rev. A62, 013402 (2000).

[17] U. Fano, Phys. Rev. A 32, 617 (1985). 